\title{
Electrical contacts to single nanowires: a scalable method allowing multiple devices on a chip. Application to a single nanowire radial $p-i-n$ junction
}

\author{
Pierre Blanc, Martin Heiss, \\ Carlo Colombo, Anna Dalmau Mallorquì \\ and Tina Saberi Safaei
}

Laboratory of Semiconductor Materials (LMSC),

Ecole Polytechnique Fédérale de Lausanne,

Lausanne, Switzerland

Email: pierre.blanc@epfl.ch

Email: martin.heiss@epfl.ch

Email: carlo.colombo@epfl.ch

Email: anna.dalmaumallorqui@epfl.ch

Email: tina.saberisafaei@epfl.ch

\section{Peter Krogstrup and Jesper Nygård}

\author{
Nano-Science Center, \\ Niels Bohr Institute, \\ University of Copenhagen, \\ 2100 Copenhagen, Denmark \\ Email: krogstrup@fys.ku.dk \\ Email:nygard@fys.ku.dk
}

\author{
Anna Fontcuberta i Morral* \\ Laboratory of Semiconductor Materials (LMSC), \\ Ecole Polytechnique Fédérale de Lausanne, \\ Lausanne, Switzerland \\ Email: anna.fontcuberta-morral@epfl.ch \\ *Corresponding author
}

\begin{abstract}
Semiconductor nanowires are currently at the forefront of research in the areas of nanoelectronics and energy conversion. In all these studies, realising electrical contacts and statistically relevant measurements is a key issue. We propose a method that enables to contact hundreds of nanowires on a single wafer in an extremely fast electron beam lithography session. The method is applied to nanowire-based radial GaAs p-i-n junction. Currentvoltage characteristics are shown, along with scanning photocurrent mapping.
\end{abstract}

Keywords: nanotechnology; electrical contacts; nanowires; solar cell; radial p-i-n junction; GaAs. 
Reference to this paper should be made as follows: Blanc, P., Heiss, M., Colombo, C., Mallorquì, A.D., Safaei, T.S., Krogstrup, P., Nygård, J. and Morral, A.F. (2013) 'Electrical contacts to single nanowires: a scalable method allowing multiple devices on a chip. Application to a single nanowire radial p-i-n junction', Int. J. Nanotechnology, Vol. 10, Nos. 5/6/7, pp.419-432.

Biographical notes: Pierre Blanc graduated with a Master's degree in Materials Science from the National Institute of Applied Sciences (INSA) of Lyon (France) in 2010. He then worked for 18 months at EPFL in the group of Professor Anna Fontcuberta i Morral on the development and characterisation of GaAs nanowire-based solar cells.

Martin Heiss was a postdoctoral researcher from 2010 till 2012 at EPFL in the group of Professor Anna Fontcuberta i Morral. He studied Physics at the Technical University Munich where he obtained his $\mathrm{PhD}$ in 2010 with a thesis titled 'Growth and properties of low-dimensional III-V semiconductor nanowire heterostructures'.

Carlo Colombo studied Physics at the University of Milano Bicocca. In 2012 he obtained his PhD from EPFL in the group of Professor Anna Fontcuberta $\mathrm{i}$ Morral with a thesis titled: 'Core-shell GaAs nanowire p-n Junctions from growth to photovoltaic and optoelectronic applications'.

Anna Dalmau Mallorquì since 2010 is doing her PhD at EPFL in the area of nanowire-based solar cells in the group of Professor Anna Fontcuberta i Morral. She studied Industrial Engineering at the Polytechnic University of Catalonia.

Tina Saberi Safaei is currently a master student in Materials Science and Engineering Section at EPFL. She obtained her bachelor's degree from Universtiy of Tehran, Iran, in 2010. Her field of interest is growth and characterisation of semiconductor nanowires.

Jesper Nygard is Professor of Experimental Physics at the Niels Bohr Institute, Copenhagen. He is Head of the Condensed Matter Section and his personal research group focuses on quantum phenomena in low-dimensional systems such as carbon nanotubes and semiconductor nanowires.

Peter Krogstrup obtained his master's degree in nanophysics at the Niels Bohr Institute, University of Copenhagen in 2009. He obtained his $\mathrm{PhD}$ in October 2012 entitled 'Dynamics of III-V nanowire growth, theory and experiments' under the supervision of Professor Jesper Nygård.

Anna Fontcuberta i Morral leads the Laboratory of Semiconductor Materials at the Institute of Materials of EPFL since 2008. She holds a PhD in Materials Science from Ecole Polytechnique (France) from 2001 and a Physics Diploma degree from the University of Barcelona (1997). Before that, she was Marie Curie Excellence grant team leader at the TU Munich, where she started her work on III-V nanowires. Before that, she was a Postdoc with Harry Atwater at the California Institute of Technology, co-founded the start-up company Aonex Technologies in the area of heterogeneous integration for multi-junction solar cells.

This paper is a revised and expanded version of a paper entitled 'Semiconductor nanowires for third generation photovoltaics' presented at the 'Nanoscale Science and Technology (NS\&T'12) Conference', Hammamet, Tunisia, 17-19 March 2012. 


\section{Introduction}

Semiconductor nanowires have been exciting interest in the last ten years for their novel physical properties and their promising applications in different fields such as nanoelectronic devices and high mobility field effect transistors [1-3]. In the last few years, part of the scientific and technological interest in nanowires has been moved to energy harvesting such as photovoltaic [4-13], thermoelectric devices [14-17] and batteries [18-22]. Most of these devices are linked to the external world via electrical contacts. Given the small size of nanowires, the overall area interfacing the contacts and the semiconductor is extremely small (few $100 \mathrm{~nm}$ or less). Variability on the sample quality can also be an issue in nanoscale materials (one doping atom more can make a large difference in the doping density). All these elements can lead to significant variations in the quality of the interface. For this reason, it has been recognised that realising statistics on the devices is extremely important. Nowadays numerous contacting procedures have been adjusted to the nature of nanoscale materials and nanowires [2327]. Most of them imply the contacting step of one device at a time. Procedures developed for contacting large ensembles of randomly positioned nanodevices on a substrate with electron beam lithography have not been published. Other approaches in the literature to place many nanowires in parallel in a controlled way on wafer scale to pre-patterned sites include dielectrophoresis [28-32] and contact printing [33].

In this paper, we present a method to design and fabricate numerous nanowire-based devices on a single step and in a parallel manner. The method is based on a user-friendly MATLAB routine that enables the fast and prompt design of many nanoscale contacts in one chip. In Section 2, we discuss the fabrication process of the electrical contacts. Section 3 introduces the automatic process of contacts design. The performance of the proposed method is explained in Section 4. We apply the method to contact nanowire GaAs radial p-i-n junctions. Result is given in Section 5. Finally, we will make a conclusion of our study in Section 6.

\section{Experimental details}

\subsection{Nanowire growth}

GaAs nanowire-based p-i-n structures were grown by Molecular Beam Epitaxy (MBE) on a silicon substrate. The core was obtained by the self-catalysed Ga-assisted process (VLS) [34-37], with a nominal gallium growth rate of $0.27 \mu \mathrm{m} / \mathrm{h}$, for $45 \mathrm{~min}$, at $T=630^{\circ} \mathrm{C}$ and $\mathrm{V} / \mathrm{III}$ beam equivalent flux ratio of 60 . The p-doping was achieved with a beryllium flux corresponding to a doping level of $3.5 \times 10^{19}$ atoms $/ \mathrm{cm}^{3}$ for planar growth [38]. The core diameter was about $120 \mathrm{~nm}$. After the growth of the p-type core, the growth conditions were switched from axial to radial growth as described elsewhere [39-41]. The shell consisted of a $30 \mathrm{~nm}$ layer without any intentional impurity, followed by $60 \mathrm{~nm}$ n-doped, using silicon as a dopant, with a nominal concentration of $5 \times 10^{18}$ atoms $/ \mathrm{cm}^{3}$. The overall diameter of the final structure is about $300 \mathrm{~nm}$ while the length is approximately $10 \mu \mathrm{m}$. 


\subsection{Contacting procedure}

The devices were prepared by transferring nanowires onto an oxidised Si substrate with a pattern having macroscopic contacts. The electrical contacts to the $\mathrm{p}$ and $\mathrm{n}$ part were realised in a double-step Electron Beam Lithography (EBL) procedure, as it will be explained in the following. We start by describing the structure of the macroscopic mask realised on a 4 inch oxidised $\mathrm{Si}$ wafer (see Figure 1). It is defined by optical lithography, followed by evaporation and lift-off. The pattern is divided into $6 \times 6$ fields. Each field is divided into $8 \times 8$ sub-fields. Each of these sub-fields contains a separate contacting region formed by a $100 \times 100 \mu \mathrm{m}$ field surrounded by macroscopic contacts. The latter corresponds to the contacts to the 'outside world' for the electrical measurements. They can be used for wire-bonding or measuring in a probe-station. More information can be found elsewhere [42]. A schematic drawing of the steps followed to contact nanowires is shown in Figure 2. After fabrication of the macroscopic contacts, the nanowires are removed from the growth substrate and dispersed in isopropyl alcohol by ultrasonication of a piece of sample in the liquid. After that, the nanowires are deposited on the patterned substrate by a pipette on the contact areas. Few droplets of the solution are enough to deposit hundreds of nanowires. After evaporation of the solution they are localised on the patterned substrate. Several methods can be applied to find the coordinates of the nanowires. If the nanowires are longer than about $3 \mu \mathrm{m}$, an optical microscope can easily image them. If the structures are smaller, scanning electron microscope is another option. Still, all images of the same run must be taken with the same magnification. After this, we use our software Autocontact [42] to treat the images, to detect the nanowires on the substrate and to define the coordinates with respect to the alignment markers closest to the nanowire.

Figure 1 Structure of the macro-contact pattern on the wafer (see online version for colours)

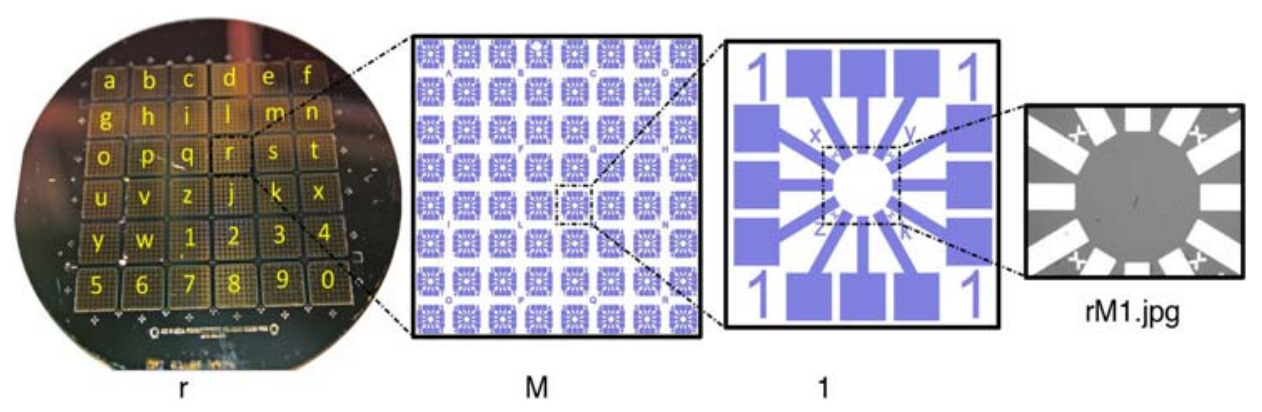

The software reproduces the position of the nanowire on the CAD drawing of the lithography mask of the contacting field. The software can further be employed to define the contacts on the nanowire. This procedure is repeated with all images containing nanowires. At the end, the software produces a file that will be read by the e-beam lithography machine to write the contacts on the resist. In order to contact a radial p-i-n junction, first we cover the sample with resist and expose one side of the nanowire. Subsequently, the $\mathrm{n}$-shell of this side of the wire is etched in a solution of $1 \mathrm{~g}$ citric acid mono-hydrate: $51 \mathrm{ml} \mathrm{H} 2 \mathrm{O}: 2 \mathrm{ml} \mathrm{H}_{2} \mathrm{O}_{2}$. In a subsequent electron beam lithography step, we write the second contact on the nanowire and evaporate both contacts formed by Pd-Ti-Au $(70 / 10 / 120 \mathrm{~nm})$. Additional details on the contacting procedure and device geometry can be found elsewhere $[41,42]$. 
Figure 2 Sketch of the steps followed during the fabrication processes (see online version for colours)
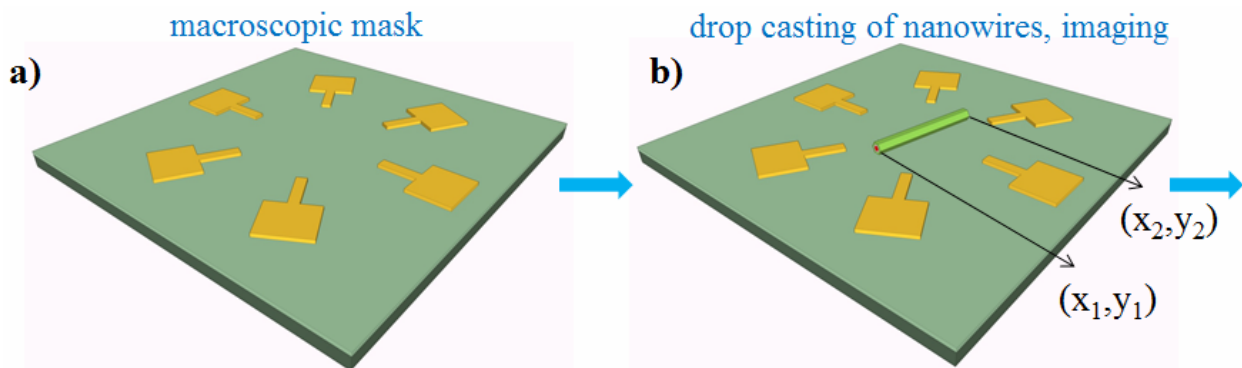

automatic generation of ebeam pattern

c)
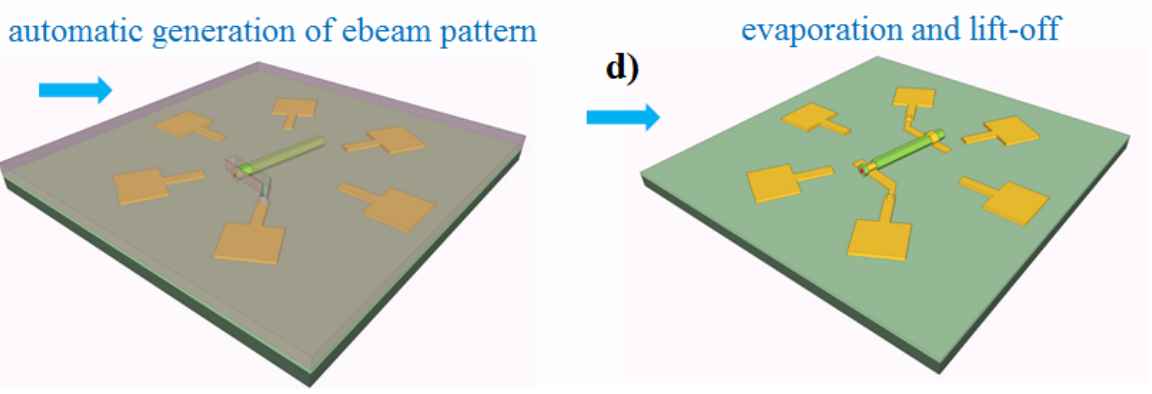

\section{Nano-contacts automation}

We have written a software to facilitate the procedure of contacting several tens to hundreds of nanowires at once [42]. The goal is to achieve enough statistics with the devices, extremely important in general in science but especially when dealing with nanoscale materials. The software is aimed to reduce the time spent on the design of the contacts. For simplicity, the program is a graphical user interface that allows the users to work directly with images (see Figure 3). This allows to design the contact for several hundreds nanowires in less than an hour.

The first step is to take pictures of the desired nanowires inside the four crosses markers (see Figure 1) with the proper magnification and good contrast between the gold and the oxidised silicon surface. The pictures can directly be named so that the filenames correspond to the location coordinate of the image inside the pattern. The first small letter corresponds to the location of the big square. The second capital letter and the third number correspond to the location of the smallest pattern inside the big square (see Figure 1).

Then the user must select the input directory (where the images are) and the output directory (where to save the processed files). By clicking on 'Run the fitting' the first picture is appearing for the calibration. The user must click on two next cross markers. The program search for the scale, rotation and translations of the images compared to a model. When the proper rotation and translations have been found, the process is terminated. The coordinates of the markers are defined making the localisation of the nanowire possible. One can control the results inside the folder 'FittedImages' in the output directory. On the right part of the software, one can adjust the parameters of the contacts (width, space, type, etc.). 
Figure 3 Graphical user interface of the AUTOCONTACT program [42] (see online version for colours)

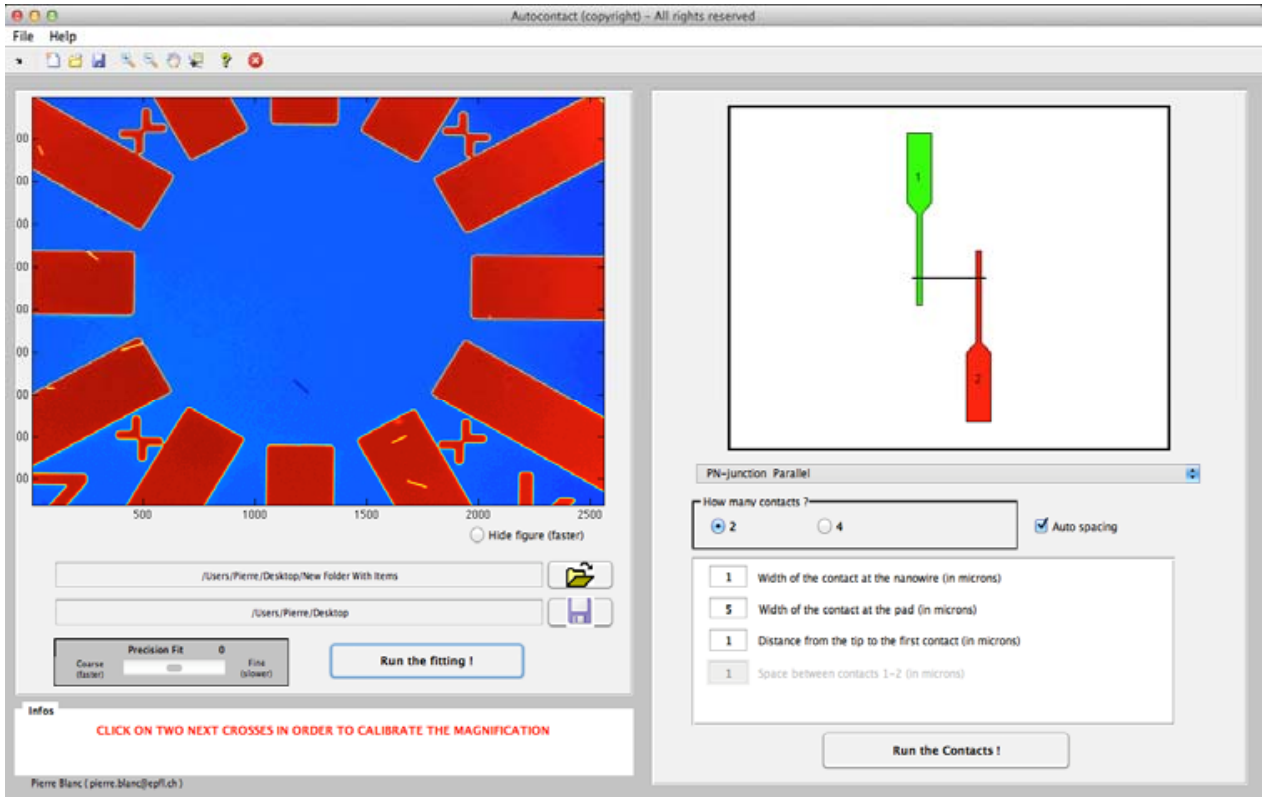

After setting all the parameters and by simply clicking on 'Run the Contacts', each image appears and allows the user to click on both sides of the nanowires to be contacted. Depending on the parameters set, the contacts will be placed automatically. It is then possible to see the resulted dxf files as well as the setting parameters in the output directory. The current version brings the possibilities to choose between a single-step EFL, a double-step EFL for pn-junctions, as well as two, four until eight contacts. If 'eight contacts' is selected, only the contacts that are on the nanowire will be drawn (the contacts outside will be deleted). The thickness, spacing and shape are all settings that can be set. Finally, there is the possibility to adjust the spacing automatically depending on the length of the nanowires.

\section{The theory behind the MATLAB code}

The program is coded with MATLAB. The following describes how the code is working. A loop lists all the images stored inside the input directory. All the images are then converted in binary colour using the greythresh function. This function chooses the threshold to minimise the intraclass variance of the black and white pixels based on Otsu's method. Every pixel having a value higher than the threshold is reset to 1 . All the others are reset to 0 .

The coordinates of each square in the pattern have been implemented so that, the name of the file gives approximately the place where the image was taken. Since it is supposed that all images have been taken with the same magnification, the first image is taken for calibration. It is asked to click on two next markers via the ButtonDownFen function. The factor converting the number of pixels separating the two points clicked, 
into the real distance, is set. An approximate scale is then defined. At this step, the program has information on the coordinates and the scale of the pictures. However, these factors are not accurate and nothing is known about the rotation and the translations. In order to retrieve the real coordinates, the program uses two fitting processes. A model that reproduces the design of the optical lithography patterns will be adjusted on the pictures until both match. The model image function depends on a four-dimensional parameter $P$.

$$
P=[\Delta x, \Delta y, \varphi, s]
$$

where $\Delta x$ and $\Delta y$ gives the translational offset from the centre as fraction of the image size, $\varphi$ is the rotation angle and $s$ is a scale correction with respect to the rough scale selected in the user interface as described above.

The model represents the smallest entities of the pattern, which are the markers and the 12 branches. It was previously created in the layout software and saved in $\mathrm{dxf}$ files. The coordinates of this pattern are then extracted and stored in a matrix. The program uses the function poly2mask that computes a binary region of interest, to create the mask.

\subsection{Coordinates extraction}

In order to fit the model with the images, the matrix representing the model and the matrix representing one of the images are compared using an XOR function: if the two same pixels from the two matrices are equal, the result would be zero. Since the two images have to be exactly superposed, the goal is to achieve a matrix filled with zeros.

The first fitting process uses the simulated annealing algorithm [43]. The simulated annealing is an application of the Metropolis-Hastings algorithm to optimisation. It is an analogy with annealing in metallurgy, involving heating and controlled cooling of a material to reduce its defects. In our case, the mask-model will be moved, rotated and rescaled with four random unknown parameters that will be refined every iteration. The extent of the search depends on a transition probability with a scale proportional to the 'temperature'. If the new solution is better than the previous, the next state is automatically accepted. Otherwise, it is accepted with a certain probability. Since the raise of the objective function can be accepted, the algorithm avoids being trapped in a local minimum.

The simulated annealing algorithm is used on the full images resized by a factor defined by the user. A smaller size reduces the computation time but decreases the accuracy. The starting points are set to zero except the scale factor, which is equal to the calibration parameter. A maximum of iteration is set to 250 . The constraints for the two translational degrees of freedom are set in order to limit the four markers to move outside the image. For the model scaling, the constraint is set to a $5 \%$ change and the maximum allowed model rotation is limited to $4.5^{\circ}$.

During acquisition of the optical microscope images, it can be easily achieved for the image to fall within these constraints. For a better refinement, another fit is performed by taking the final results as starting points. However, not the full picture will be taken, but only the four markers (not resized) and will be summed up. The fitting algorithm uses the patternsearch function. This refinement allows the model to move with a tolerance of $\pm 0.05 \mathrm{P}$. 


\subsection{Design of contacts}

The two fitting processes allow retrieving parameter $P$ that defines the coordinate transformation between the microscope image and the mask layout (see Figure 4b). The missing part is now the design of contacts. The precise position and rotation of the nanowire are calculated when the user clicks on both ends of the nanowire in the microscope image using the coordinate transformation obtained by the fit. A small rectangular portion of the contacts, with the geometrical characteristics that can be defined by the user, is drawn perpendicularly to the NW. The direction of this portion is represented by the red axis in Figure 5. Depending on the position of the NW, this portion must be bounded to one of the pad represented by the letter $c_{i}$. The contacts should be as straight as possible and they should not cross either each other, or the nanowire.

Figure 4 Fitting principle used by our AUTOCONTACT program (see online version for colours)

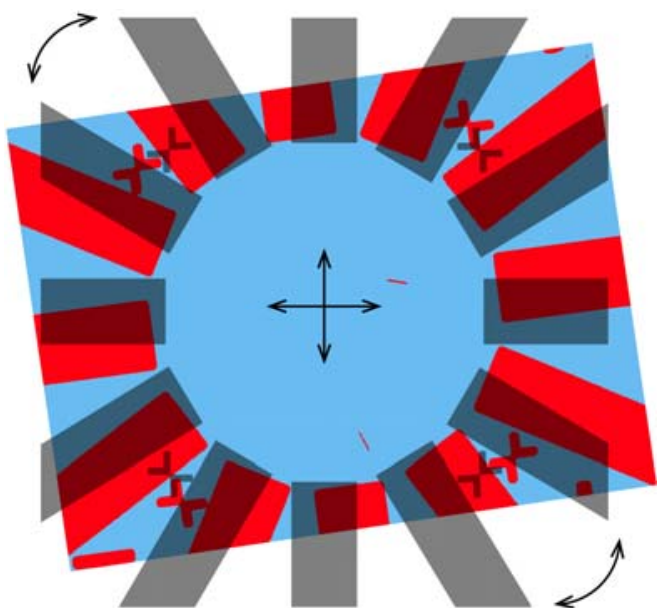

(a) Fitting on process (in grey, the mask moving to superpose the image)

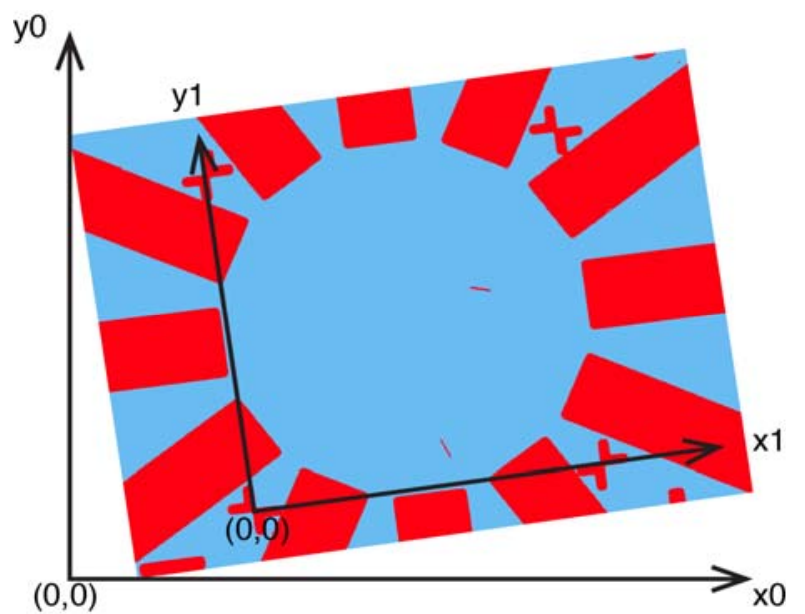

(b) After fitting, a set of coordinate is define 
Figure 5 Schematics showing how the contacts are designed, once the position of the nanowires is known (see online version for colours)

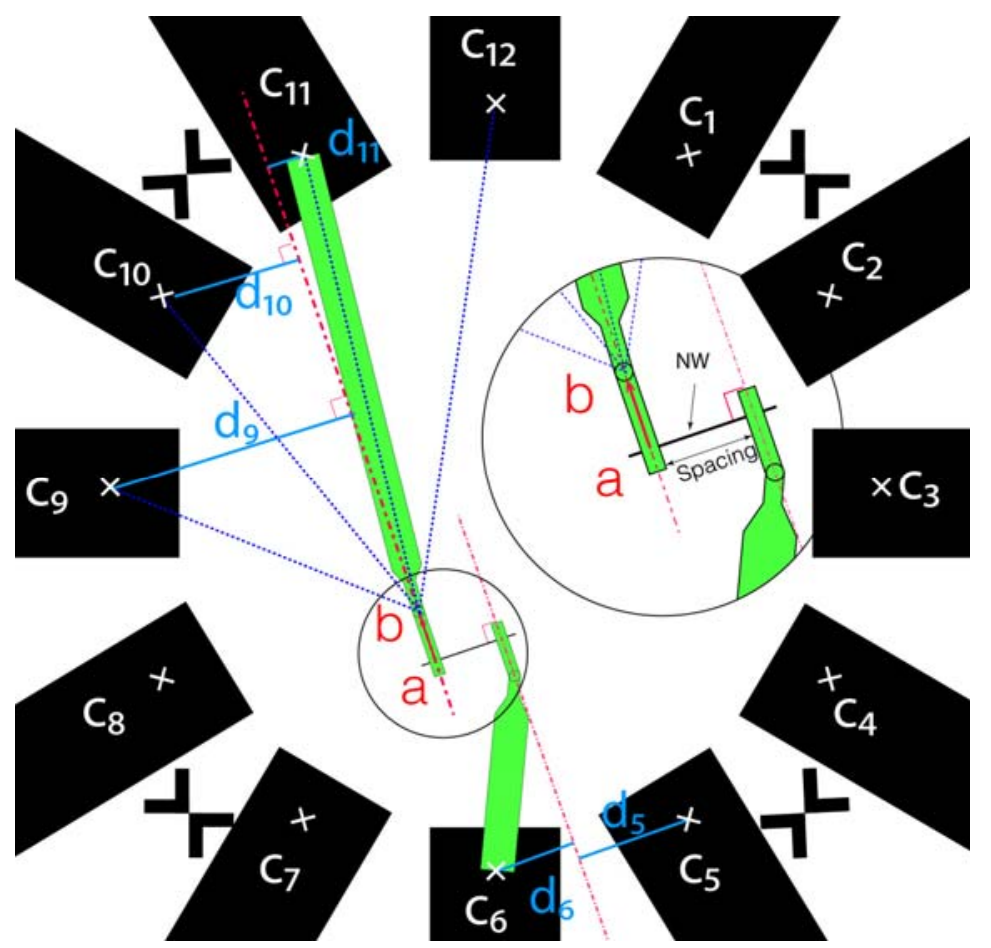

The best way to solve this is to find the shortest perpendicular distance between the axis of the portion contact (red axis in Figure 5) and the pad $c_{i}$. The following equations are solved:

$$
d_{i}\left\{\begin{array}{cl}
\frac{\overrightarrow{a b} \wedge \overrightarrow{b c_{i}}}{\|\overrightarrow{a b}\|} & \text { if } \overrightarrow{a b} \cdot \overrightarrow{b c_{i}} \geq 0 \\
\theta & \text { if } \overrightarrow{a b} \cdot \overrightarrow{b c_{i}}<0
\end{array}\right.
$$

The perpendicular distances to the red axis are found via the cross product. The conditions state that the pads chosen must be in the same direction as the vector $\overrightarrow{a b}$. This avoids the issue of having contacts with an angle higher than $90^{\circ}$ with the first portion drawn. The same procedure is done for the second contact but in the opposite direction. When there are more than two contacts, the two inner ones are chosen for respecting the equations. The other contacts are then defined on the pads beside.

Once the paths are defined, the coordinates and the shape of the contacts will be added line by line on the dxf output file containing the electron beam lithography pattern. 


\section{Results}

To demonstrate the performance of electron beam lithography contacted nanodevice, we now turn to our results on radial nanowire p-i-n-junction operated in light absorption. We have studied the properties of the radial junction by means of photo-current mapping of our nanowires. The sample is placed on an $\mathrm{x}-\mathrm{y}$ piezo-driven scanning stage and a focused laser spot with a diameter in the order of $1 \mu \mathrm{m}$ is scanned over an area of $15 \times 15 \mu \mathrm{m}$. Meanwhile the short circuit current is acquired. The laser wavelength is $520 \mathrm{~nm}$ and the intensity of incident beam is approximately $2.5 \mathrm{nW}$. Moreover we modulate the laser light with a chopper wheel allowing measuring the photo-current with a lock-in amplifier.

Figure 6 (a) I-V characteristics of a nanowire radial p-i-n junction device. (b) Lock-in photocurrent mapping of the nanowire device illuminated with a $520 \mathrm{~nm}$ wavelength. The device contacts that cover part of the nanowire are schematically indicated by the white lines (see online version for colours)
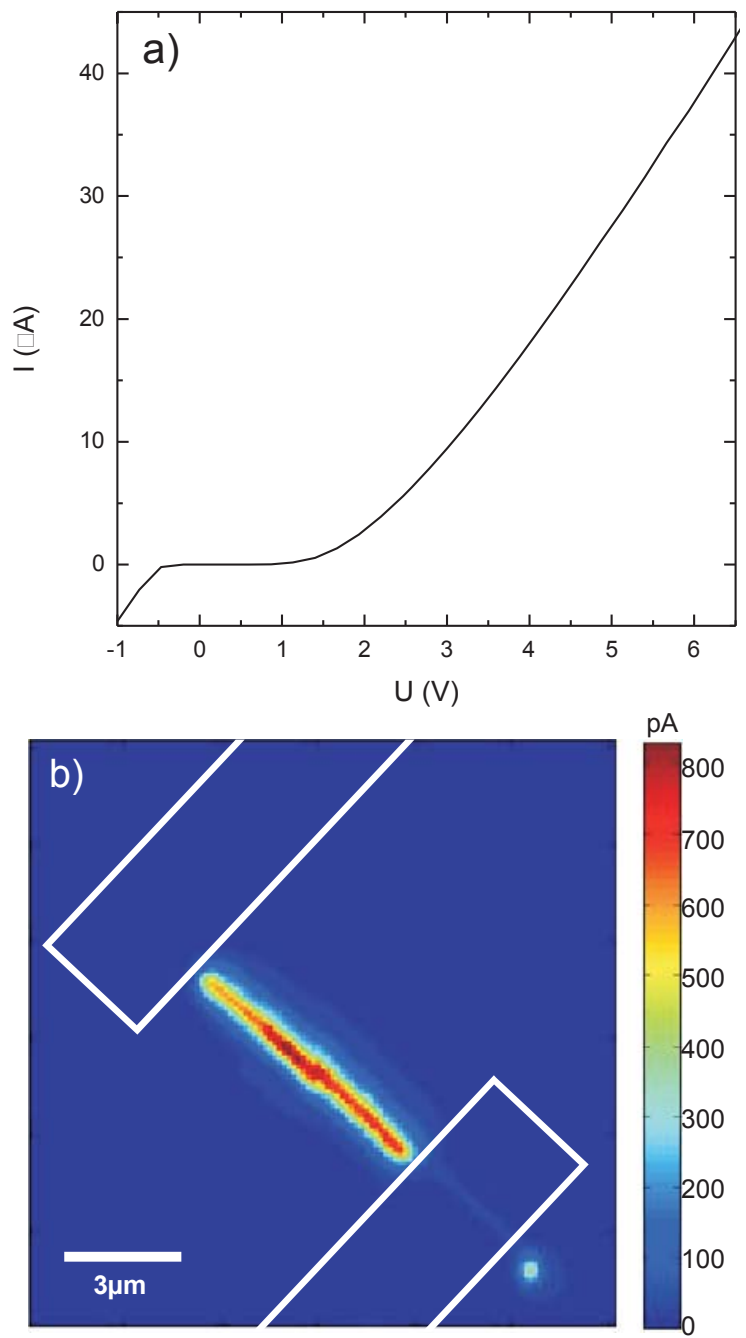
The I-V characteristics of a single nanowire device are shown in Figure 6 together with the spatial mapping of laser beam induced photo-current in the same nanowire. The photo-current is generated over the entire length of the exposed radial p-i-n junction indicating the good quality of the device.

The I-V curve in Figure 6a can be modelled with a diode equation and series resistance:

$$
I=I_{s} \exp \frac{q\left(U-R_{s} I\right)}{n k_{b} T}
$$

A fit to the experimental data allows to obtain a value for the series resistance of $R=104 \mathrm{k} \Omega$.

\section{Conclusion}

In conclusion we have presented a technique that allows the contacting of multiple single nanowires that are randomly oriented on a host substrate with an automatised and scalable approach. This allows to significantly increase the production throughput and to obtain more statistical information on the properties of the nanowires. One should point out that our approach is easily transferable on other nanoscale materials such as exfoliated graphene or carbon nanotubes. Indeed the advantage of sample preparation will help in the future to further improve characterisation and understanding of nanoscale materials.

\section{Acknowledgements}

The authors thank funding of ERC through grant UpCon and SNF funding through grants 121758/1 and 129775/1/ and the NCCR QSIT. PK and JN thank financial support by the Danish National Advanced Technology Foundation through project 022-2009-1.

\section{References}

1 Wallentin, J., Persson, J.M., Wagner, J.B., Samuelson, L., Deppert, K. and Borgström, M.T. (2010) 'High-performance single nanowire tunnel diodes', Nano Letters, Vol. 10, No. 3, pp.974-979.

2 Xiang, J., Lu, W., Hu, Y., Wu, Y., Yan, H. and Lieber, C.M. (2006) 'Ge/Si nanowire heterostructures as high-performance field-effect transistors', Nature, Vol. 441, No. 7092, pp.489-493.

3 Mourik, V., Zuo, K., Frolov, S.M., Plissard, S.R., Bakkers, E.P.A.M. and Kouwenhoven, L.P. (2012) 'Signatures of majorana fermions in hybrid superconductor-semiconductor nanowire devices', Science, Vol. 336, No. 6084, pp.1003-1007.

4 Bakkers, E.P.A.M., Dam, J.A.V., De Franceschi, S., Kouwenhoven, L.P., Kaiser, M., Verheijen, M., Wondergem, H. and Sluis, P.V.D. (2004) 'Epitaxial growth of InP nanowires on germanium', Nature Materials, Vol. 3, No. 11, pp.769-773.

5 Martensson, T., Svensson, C.P.T., Wacaser, B.A., Larsson, M.W., Seifert, W., Deppert, K., Gustafsson, A. and Wallenberg, L.R. (2004) 'Epitaxial III-V nanowires on silicon', Nano Letters, Vol. 4, No. 10, pp.1987-1990. 
6 Tian, B., Kempa, T.J. and Lieber, C. M., (2009) 'Single nanowire photovoltaics', Chemical Society Reviews, Vol. 38, No. 1, pp.16-24.

7 Czaban, J.A., Thomposon, D.A. and LaPierre, R.R. (2009) 'GaAs core-shell nanowires for photovoltaic applications', Nano Letters, Vol. 9, No. 1, pp.148-154.

8 Kelzenberg, M.D., Boettcher, S.W., Petykiewicz, J.A., Turner-Evans, D.B., Putnam, M.C., Warren, E.L., Spurgeon, J.M., Briggs, R.M., Lewis, N.S. and Atwater, H.A. (2010) 'Enhanced absorption and carrier collection in Si wire arrays for photovoltaic applications', Nature Materials, Vol. 9, No. 4, pp.239-244.

9 Boettcher, S.W., Spurgeon, J.M., Putnam, M.C., Warren, E.L., Turner-Evans, D.B., Kelzenberg, M.D., Maiolo, J.R., Atwater, H.A. and Lewis, N.S. (2010) 'Energy-conversion properties of vapor-liquid-solid-grown silicon wire-array photocathodes', Science, Vol. 327, No. 5962, pp.185-187.

10 Colombo, C., Heiß, M., Gratzel, M. and Fontcuberta i Morral, A. (2009) 'Gallium arsenide pi-n radial structures for photovoltaic applications', Applied Physics Letters, Vol. 94, No. 17, pp. 173108 .

11 Dalmau-Mallorqui,A., Epple, F.M., Fan, D., Demichel, O. and Fontcuberta i Morral, A. (2012) 'Effect of the pn junction engineering on Si micro-pillar array solar cells', Physica Status Solid A, Vol. 208, No. 8, pp.1588-1591.

12 Wallentin, J. et al. (2013) 'InP nanowire array solar cells achieving $13.8 \%$ efficiency by exceeding the ray optics limit', Science, Vol. 339, No. 6123, pp.1057-1060.

13 Krogstrup, P. et al. (2013) 'Single-nanowire solar cells beyond the Shockley-Queisser limit', Nature Photonics, Vol. 7, pp.306-310.

14 Hochbaum, A.I., Chen, R., Delgado, R.D., Liang, W., Garnett, E.C., Najarian, M., Majumdar, A. and Yang, P., (2008) 'Enhanced thermoelectric performance of rough silicon nanowires', Nature, Vol. 451, No. 7175, pp.163-167.

15 Yu, J.K., Mitrovic, S., Tham, D., Varghese, J. and Heath, J.R. (2010) 'Reduction of thermal conductivity in phononic nanomesh structures', Nature Nanotechnology, Vol. 5, No. 10, pp.718-721.

16 Galli, G. and Donadio, D. (2010) 'Thermoelectric materials: silicon stops heat in its track', Nature Nanotechnology, Vol. 5, No. 10, pp.701-702.

17 Lin, W.W., Hehn, M., Chaput, L., Negulescu, B., Andrieu, S., Montaigne, F. and Mangin, S. (2012) 'Giant spin-dependent thermoelectric effect in magnetic tunnel junctions', Nature Communications, Vol. 3, p.774.

18 Chan, C.K., Peng, H., Liu, G., McIlwrath, K., Zhang, X. F., Huggins, R.A. and Cui, Y. (2008) 'High-performance lithium battery anodes using silicon nanowires', Nature Nanotechnology, Vol. 3, No. 1, pp.31-35.

19 Chan, C.K., Zhang, X.F. and Cui, Y. (2008) 'High capacity Li ion battery anodes using Ge nanowires', Nano Letters, Vol. 8, No. 1, pp.307-309.

20 Ge, M.Y., Rong, J.P., Fang, X. and Zhou, C.W. (2012) 'Porous doped silicon nanowires for lithium ion battery anode with long cycle life', Nano Letters, Vol. 12, No. 5, pp.2318-2323.

21 Yang, H., Huang, S., Huang, X., Fan, F.F., Liang, W.T., Liu, X.H., Chen, L.Q., Huang, J.Y., Li, J., Zhu, T. and Zhang, S.L. (2012) 'Orientation-dependent intefacial mobility governs the anisotropic swelling in lithiated silicon nanowires', Nano Letters, Vol. 12, No. 4, pp.1953-1958.

22 Guo, W.X., Xue, X.Y., Wang, S.H., Lin, C.J. and Wang, Z.L. (2012) 'An integrated power pack of Dy-sensitized solar cell and Li battery based on double-sided TiO2 nanotube arrays', Nano Letters, Vol. 12, No. 5, pp.2250-2523.

23 Cui, Y. and Lieber, C.M. (2001) 'Functional nanoscale electronic devices assembled using silicon nanowire building blocks', Science, Vol. 291, No. 5505, pp.851-853. 
24 Li, X., Wang, X., Zhang, L., Lee, S. and Dai, H. (2008) 'Chemically derived, ultrasmooth graphene nanoribbon semiconductors', Science, Vol. 319, No. 5867, pp.1229-1232.

25 Dufouleur, J., Colombo, C., Garma, T., Ketterer, B., Uccelli, E., Nicotra, M. and Fontcuberta i Morral, A., (2010) 'P-doping mechanisms in catalyst-free gallium arsenide nanowires', Nano Letters, Vol. 10, No. 5, pp.1734-1740.

26 Thunich, S., Prechtel, L., Spirkoska, D., Abstreiter, G., Morral, A.F.I. and Holleitner, A.W. (2009) 'Photocurrent and photoconductance properties of a GaAs nanowire', Applied Physics Letters, Vol. 95, No. 8, p.083111.

27 Prechtel, L., Padilla, M. Erhard, N., Karl, H. Abstreiter, G. Morral, A.F.I. and Holleitner, A.W. (2012) 'Time-resolved photoinduced thermoelectric and transport currents in GaAs nanowires', Nano Letters, Vol. 12, No. 5, pp.2337-2341.

28 Hermanson, K.D., Lumsdon, S.O., Williams, J.P., Kaler, E.W. and Velev, O.D. (2001) 'Dielectrophoretic assembly of electrically functional microwires from nanoparticle suspensions', Science, Vol. 294, No. 5544, pp.1082-1086.

29 Raychaudhuri, S., Dayeh, S.A., Wang, D. and Yu, E.T. (2009) 'Precise semiconductor nanowire placement through dielectrophoresis', Nano Letters, Vol. 9, No. 6, pp.2260-2266.

30 Lao, C.S., Liu, J., Gao, P.X., Zhang, L.Y., Davidovic, D., Tummala, R. and Wang, Z.L. (2006) ' $\mathrm{ZnO}$ nanobelt/nanowire Schottky diodes formed by dielectrophoresis alignment across Au electrodes', Nano Letters, Vol. 6, No. 2, pp.263-266.

31 Duchamp, M., Lee, K., Dwir, B., Seo, J.W., Kapon, E., Forro, L. and Magrez, A. (2010) 'Controlled positioning of carbon nanotubes by dielectrophoresis: insights into the solvent and substrate role', ACS Nano, Vol. 4, No. 1, pp.279-284.

32 Freer, E.M., Grachev, O., Duan, X., Martin, S. and Stumbo, D.P. (2010) 'High-yield selflimiting single-nanowire assembly with dielectrophoresis', Nature Nanotechnology, Vol. 5, No. 7, pp.525-530.

33 Fan, Z., Ho, J.C., Jacobson, Z.A., Yerushalmi, R., Alley, R.L., Razavi, H. and Javey, A. (2008) 'Wafer-scale assembly of highly ordered semiconductor nanowire arrays by contact printing', Nano Letters, Vol. 8, No. 1, pp.20-25.

34 Fontcuberta i Morral, A., Colombo, C., Abstreiter, G., Arbiol, J. and Morante, J.R. (2008) 'Nucleation mechanism of gallium-assisted molecular beam epitaxy growth of gallium arsenide nanowires', Applied Physics Letters, Vol. 92, No. 6, p.063112.

35 Colombo, C., Spirkoska, D., Frimmer, M., Abstreiter, G. and Fontcuberta i Morral, A. (2008) 'Ga-assisted catalyst-free growth mechanism of GaAs nanowires by molecular beam epitaxy', Physical Review B, Vol. 77, No. 15, p.155326.

36 Uccelli, E., Arbiol, J., Magen, C., Krogstrup, P., Russo-Averchi, E., Heiss, M., Mugny, G., Morier-Genoud, F., Nygard, J., Morante, J.R. and Fontcuberta i Morral, A. (2011) 'Threedimensional multiple-order twinning of self-catalyzed GaAs nanowires on Si substrates', Nano Letters, Vol. 11, No. 9, pp.3827-3832.

37 Russo-Averchi, E., Heiss, M., Michelet, L., Krogstrup, P., Nygard, J., Magen, C., Morante, J.R., Uccelli, E., Arbiol, J. and Fontcuberta I Morral, A. (2012) 'Suppression of three dimensional twinning for a $100 \%$ yield of vertical GaAs nanowires on silicon', Nanoscale, Vol. 4, No. 5, pp.1486-1490.

38 Casadei, A. et al. (2013) 'Doping incorporation paths in catalyst-free Be-doped GaAs nanowires', Appl. Phys. Lett., Vol. 102, No. 1, 013117.

39 Heigoldt, M., Arbiol, J., Spirkoska, D., Rebled, J.M., Conesa-Boj, S., Abstreiter, G., Peiro, F., Morante, J.R. and Fontcuberta i Morral, A. (2009) 'Long range epitaxial growth of prismatic heterostructures on the facets of catalyst-free GaAs nanowires', Journal of Materials Chemistry, Vol. 19, No. 7, pp.840-848.

40 Fontcuberta i Morral, A., Spirkoska, D., Arbiol, J., Heigoldt, H., Morante, J.R. and Abstreiter, A. (2008) 'Prismatic quantum heterostructures synthesized on molecular-beam epitaxy GaAs nanowires', Small, Vol. 4, No. 7, p.899. 
41 Colombo, C., Krogstrup, P., Nygard, J., Brongersma, M.L. and Fontcuberta i Morral, A. (2011) 'Engineering light absorption in single-nanowire solar cells with metal nanoparticles', New Journal of Physics, Vol. 13, No. 12, p.123026.

42 Blanc, P. and Heiss, M. (2012) 'Matlab based autocontact software developed at LMSC', EPFL. Available online at: http://www.mathworks.com/matlabcentral/fileexchange/36036autocontact-nanodevices

44 Kirkpatrick, S., Gelatt, C.D. and Vecchi, M.P. (1983) 'Optimization by simulated annealing', Science, Vol. 220, No. 4598, pp.671-680. 